\title{
Polyhedron Biosensor Based on Surface Plasmon Resonance Technology
}

\author{
Jyh-Shyan Chiu, ${ }^{1}$ Shinn-Fwu Wang, ${ }^{2 *}$ and Wen-June Wang ${ }^{1}$ \\ ${ }^{1}$ Department of Electrical Engineering, National Central University, Taoyuan 32001, Taiwan \\ ${ }^{2}$ Department of Electronic Engineering, Chien Hsin University of Science and Technology, Taoyuan 32097, Taiwan
}

(Received December 18, 2017; accepted January 26, 2018)

Keywords: polyhedron biosensor, surface plasmon resonance (SPR), attenuated total reflection (ATR)

In this paper, we propose a photoelectric biosensor exhibiting instantaneous and simple measurement properties. On the basis of surface plasmon resonance principles, the proposed biosensor capitalizes on the adhesion activity between test fluids and metal films. Attenuated total reflection (ATR) occurs when light permeates metal films, resulting in a fluctuation in light intensity. Thus, the fluid properties can be determined by measuring the intensity of the light that exits the fluid. Because adding chemicals or agents to the proposed biosensor is unnecessary, the properties of the measured sample are unaffected during measurements. The photoelectric biosensor features several advantages such as instantaneous measurement, high sensitivity, high resolution, simple experimental architecture, and low cost.

\section{Introduction}

In recent years, surface plasmon resonance (SPR) sensors have attracted much attention ${ }^{(1-6)}$ because they have high sensitivity for changes in the refractive index of the tested medium. As the surface plasmons are excited, a dip will appear in the plot of the reflectance of TM wave versus the incidence angle. The corresponding incidence angle of the dip is called the resonance angle. In fact, some SPR sensors have been applied to the field of biochemical sensing in the past few decades. For example, Feifer et al. proposed a real-time sensing method for specific molecular binding involving SPR spectroscopy. ${ }^{(1)}$ In addition, Jabbari et al. presented a novel enzyme-based SPR-biosensor to detect bromocriptine as an ergoline derivative drug. ${ }^{(2)}$ However, advancements in research and technology over the years have resulted in diversified development of biosensors. SPR technologies are currently the most commonly used in biosensors. Although a diverse range of biosensors are publicly available, a considerable number of biosensors are still undergoing laboratory testing and validation. With advancements in sophisticated technologies, continued effort has been invested in improving the resolution, sensitivity, accuracy, and measuring speed of biosensors. The greatest challenge currently faced by researchers is to transform such laboratory prototypes into commercial products. Therefore, this field of research is attracting growing interest.

*Corresponding author: e-mail: sfwang@uch.edu.tw

http://dx.doi.org/10.18494/SAM.2018.1858 
To achieve reasonable measurement accuracy levels, current biosensor measurement practices involve the addition of chemical reagents, which may cause adverse effects on the properties of the test substance. Because of this problem, biosensor researchers in recent years have tended to avoid using such chemical reagents. Even though several biosensors have been commercialized, most of these devices are still being tested in the laboratory.

In the current study, we propose a polyhedron biosensor based on the sensor presented by Wang et al. ${ }^{(7)}$ A BK7 strip optical prism is embedded in the proposed biosensor. The sensing unit of the prism is coated with two layers of metal films: a layer of 2-nm-thick titanium and a layer of 45.5-nm-thick gold. The proposed sensor differs from that of Wang et al. in the manner in which the properties of the medium are elucidated. Wang et al. analyzed the medium's properties by measuring the phase change; by contrast, in the current study, these properties were datermind by measuring the light intensity. Therefore, the proposed sensor considerably reduces the cost of the measurement system while maintaining a resolution of up to $10^{-6}$ orders of magnitude. This biosensor can execute measurements without chemical additives. It also features numerous advantages, such as instantaneous and rapid measurements, as well as high resolution.

\section{Principles}

It is well known that the phenomenon of SPR in thin metal films is highly sensitive to the optical and structural properties of the metal interface. When the wave vector of p-polarization light matches the SPR condition for combing the wavelength and incidence angle, surface plasmons are excited, resulting in energy loss from the reflected light. By applying SPR excitation, researchers can determine the refractive index of a sample material at a given wavelength. In fact, the reflectivity has a strong absorption dip around the resonant angle if SPR occurs. However, a considerable number of studies, as stated in Sect. 1, have been carried out on SPR in the past decades. However, the SPR technology could be applied in many fields besides the biophotonics, biochemical, and biological fields.

In this study, polygonal prism coupling was used to excite the SPR coupling configuration. Attenuated total reflection (ATR) can be achieved by applying the SPR excitation technique to a prism; ATR is a phenomenon in which light permeating through a metal layer produces resonance in the metal layer, causing the energy to decay exponentially because of energy absorption by the metal. The Kretschmann configuration was employed as the architecture for conducting the experiment.

In this study, a four-layer stacked structure coupled in the Kretschmann configuration was used. The employed structural materials include a prism (BK7), titanium (Ti) film, gold (Au) film, and test substance (Fig. 1). Dielectric constants ${ }^{(8)}$ of the material are

$$
\begin{gathered}
\varepsilon_{1}=\left(n_{1}\right)^{2}=(1.51509)^{2}, \\
\varepsilon_{2}=-6.8873+20.43 j,
\end{gathered}
$$




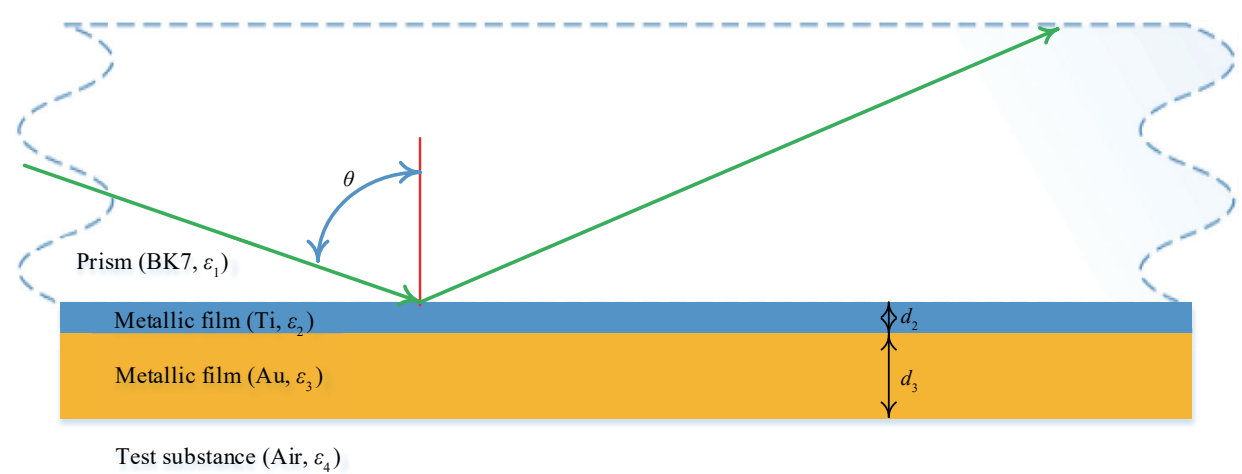

Fig. 1. (Color online) Four-layer Kretschmann configuration coupled structure.

$$
\begin{gathered}
\varepsilon_{3}=-11.892+1.2 j, \\
\varepsilon_{4}=\left(n_{4}\right)^{2} .
\end{gathered}
$$

When the incidence angle $\theta$ is equal to the resonance angle $\theta_{S P}$, resonance is generated, exciting an SPR wave. The reflection coefficient of the S-polarized and P-polarized light in ATR can be expressed as ${ }^{(9,10)}$

$$
\begin{gathered}
r_{234}^{t}=\frac{r_{23}^{t}+r_{34}^{t} e^{j 2 k_{23} d_{3}}}{1+r_{23}^{t} r_{34}^{t} e^{j 2 k_{23} d_{3}}}, \\
r_{1234}^{t}=\frac{r_{12}^{t}+r_{234}^{t} e^{j 2 k_{22} d_{2}}}{1+r_{12}^{t} r_{234}^{t}},
\end{gathered}
$$

where

$$
\begin{aligned}
r_{i j}^{t} & =\frac{E_{i}^{t}-E_{j}^{t}}{E_{i}^{t}+E_{j}^{t}}, \\
& =\left\{\begin{array}{cc}
\frac{k_{z I}}{n_{I}^{2}} & t=p \\
k_{z I} & t=s
\end{array}\right\}, I=i, j ; i, j=1,2,3 \ldots
\end{aligned}
$$

where $t$ represents $S$ - or $P$-polarization, and $d_{2}$ and $d_{3}$ in Eqs. (5) and (6) represent the thicknesses of the second and third layers of the dielectric medium, respectively.

In Eq. (8), $k_{z i(j)}$ represents the wave vector component of medium $i(j)$ along the $z$-axis, and

$$
k_{z i(j)}=k_{0} \sqrt{\left(n_{i(j)}^{2}-n_{1}^{2} \sin ^{2} \theta\right)},
$$


where $n_{1}$ is the refractive index of the prism, $n_{2}$ is the refractive index of the titanium film (Ti), $n_{3}$ is the refractive index of the gold film (Au), $n_{4}$ is the refractive index of the test substance (air), and $k_{0}$ is the wave vector of light in vacuum. The surface plasmons are excited when the wave vector of incident light, $k_{x}$, and the real part of the surface plasmon wave vector, $k_{s p}$, satisfy the matching condition given by

$$
k_{x}=k_{0} n_{1} \sin \theta=\left(\frac{2 \pi}{\lambda_{0}}\right) n_{1} \sin \theta=\operatorname{Re}\left\{k_{s p}\right\}
$$

where $k_{0}$ is the free-space wave number and $\lambda_{0}$ the wavelength of light in the free space. At this moment, $\theta=\theta_{s p}$ is the resonance angle. The surface plasmon wave vector, $k_{s p}$, can be expressed as

$$
k_{s p}=k_{0}\left[\frac{\varepsilon_{2} \varepsilon_{3}}{\varepsilon_{2}+\varepsilon_{3}}\right]^{0.5},
$$

where $\varepsilon_{2}$ and $\varepsilon_{3}$ are the wavelength-dependent complex dielectric permittivities of $\mathrm{Ti}$ and $\mathrm{Au}$ films, respectively. By combining Eqs. (10) and (11), the resonance angle $\theta_{s p}$ can be obtained.

Assuming the reflection coefficients of $S$ - and $P$-polarized light to be $r_{1234}^{S}$ and $r_{1234}^{P}$, they can be written as

$$
\begin{aligned}
& r_{1234}^{S}=\left|r_{1234}^{S}\right| e^{j \phi_{S}}, \\
& r_{1234}^{P}=\left|r_{1234}^{P}\right| e^{j \phi_{P}} .
\end{aligned}
$$

The total light intensity of the ATR is

$$
\begin{gathered}
R_{S}=\left|r_{1234}^{S}\right|^{2}, \\
R_{P}=\left|r_{1234}^{P}\right|^{2} .
\end{gathered}
$$

\section{Experimental Setup}

In the study, the sensor is designed as a polygonal prism made of BK-7 glass with a refractive index of 1.51509. The dimensions of the polyhedral prism are shown in Fig. 2. To design the

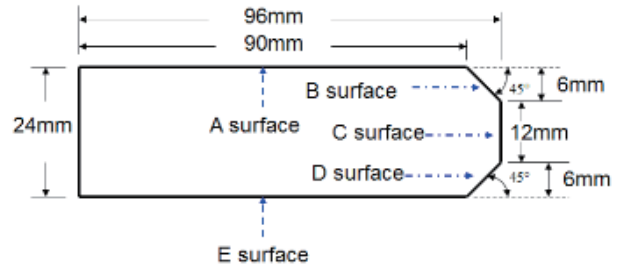

(a)

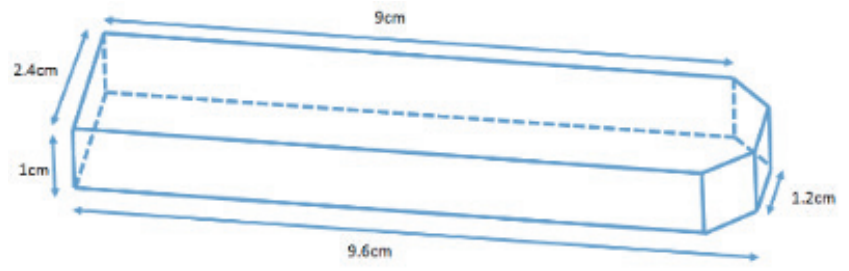

(b)

Fig. 2. (Color online) The dimensions of the sensor, (a) top and (b) 3D views. 
biosensor, we must prepare a prism with the length, width, and height of 9.6, 2.4, and $1.0 \mathrm{~cm}$, respectively. Subsequently, two $0.6 \times 0.6 \mathrm{~cm}$ triangles are cut from the corners of the prism, as shown in Fig. 2. Afterwards, the polyhedronal prism is coated with two layers of metal films, that is, a layer of 2-nm-thick titanium and a layer of 45.5-nm-thick gold, using a thermal system manufactured by Kao Duen Technology Corp. Moreover, we design a sink in which to place the prism and the tested medium. The sink is made of acrylic with the length, width, height, and thickness of $11.2,4.2,2.4$, and $0.3 \mathrm{~cm}$, respectively. A photograph of the sensor and sink is shown in Fig. 3(a). In the experiment, we fill ethanol solutions of different concentrations into the tank until the level of the solution reaches the height of $1.0 \mathrm{~cm}$. Thus, the contact area of sample solutions stays the same.

In this study, a laser diode with a wavelength of $635 \mathrm{~nm}$ is used as the light source. Figure 3(b) depicts a schematic of the polygonal prismatic biosensor and optical path. The material (BK7) is placed in a test fluid slot composed of acrylic. After passing through the air (refractive index $n_{1}=1.0003$ ), the light penetrates the acrylic fluid slot as well as the test fluid and reaches the polygonal prismatic biosensor.

To create SPR on each side of the prism when light enters the polygonal prismatic biosensor, an appropriate incidence angle is essential to achieve optimal sensitivity. To realize this, a polygonal prism was designed, and Matlab software was used to simulate the ATR intensity at various incidence angles. In fact, the dielectric constant for gold is related to wavelength. For the wavelength of $\lambda=635 \mathrm{~nm}$, the dielectric constants ${ }^{(8)}$ of gold (Au) and titanium (Ti) are $-11.892+1.2 j$ and $-6.8873+20.43 j$, respectively. Figure 4 depicts the plot of the reflectivity versus incidence angle at the metal film-dielectric interface for the refractive index in the range of 1.33-1.365 of the tested medium as the light undergoes ATR. Owing to multiple ATRs in the experiment, the test signal could be very weak if the chosen incidence angle is around the resonance angle $\left(\theta_{s p} \sim 71.7^{\circ}\right.$ as $\left.n_{4}=1.33\right)$. In this paper, the main purpose for choosing an incidence angle $\alpha_{\mathrm{i}}$ of $71.0^{\circ}$ is to allow comparison with the results of the experiments of Wang et al. Although the incidence angles at B and D surfaces are identical and equal to $64^{\circ}$, there is a slight variation of $R_{p}$ on the two surfaces. On the basis of this consideration, the incidence angle at $\mathrm{A}$ and $\mathrm{E}$ surfaces is chosen as $\alpha_{i}=71.0^{\circ}$. Using $\alpha_{i}=71.0^{\circ}$, we have the optical path shown in Fig. 3. From Fig. 3, it can be seen that the light will undergo ATR twice at the incidence angle $\alpha=71^{\circ}$ on both side surfaces A and E. It will undergo ATR once at the incidence angle $\alpha=64^{\circ}$ on both side surfaces B and D. In addition, the light undergoes ATR once at the incidence angle

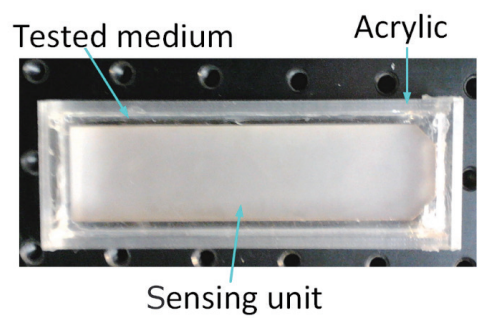

(a)

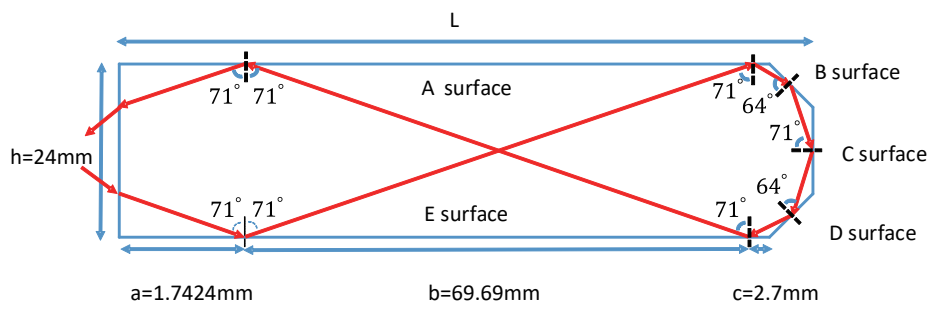

(b)

Fig. 3. (Color online) (a) Photograph of the sensor sink and (b) polygonal sensor and optical paths. 


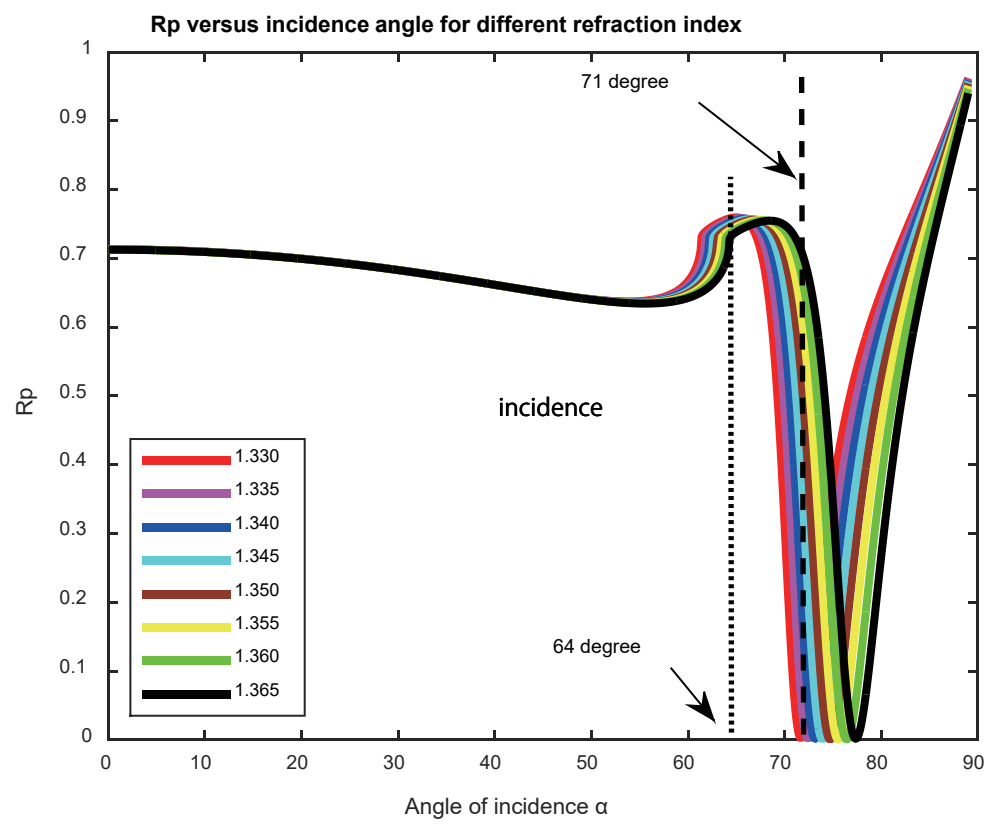

Fig. 4. (Color online) Angle versus intensity for different refractive indices at $635 \mathrm{~nm}$ (after one instance of ATR).

$\alpha=71^{\circ}$ on the end surface C. According to the statement above, the light will undergo ATR seven times before it exits the polygonal prism.

Figure 5 illustrates the architecture of the proposed biosensor. In this architecture, a laserdiode-driven circuit is used to drive the laser diode as the light source. In the experimental setup, the p-polarization light extracted from the laser light source is incident on the beam splitter (BS) and is divided into the reflected and transmitted lights. The transmitted light enters the polyhedral biosensor located at the center of a precision rotation stage (M-URMPP) with an angular resolution of $0.001^{\circ}$, manufactured by Newport. Afterwards, the light undergoes ATR multiple times in the biosensor. Subsequently, the light exiting the polyhedral biosensor is received by the test detector (OSRAM Opto Semiconductors Photodiodes BPW-21). The received signal is the test signal and its intensity is denoted as $I_{t}$. In addition, the reflected light from the BS measured by the reference detector is the test signal, and its intensity is denoted as $I_{r}$. The two signals are sent to our laboratory-made signal processing circuit simultaneously. In the experiment, two digital multimeters (GDM-8246, Good Will Instrument Co., Ltd.) are used to measure the two signals. Thus, the reflectivity $R_{p}$ of p-polarization light is given by

$$
R_{p}=\frac{I_{t}}{I_{r}} \times 100 \%
$$

After some calculations using a personal computer (PC), the refractive index of the tested medium can be obtained. The laser light traveling in the polygonal prismatic biosensor undergoes ATR seven times before reaching the detector, which is used to measure changes in light intensity. A photograph of the experimental setup is shown in Fig. 6. 


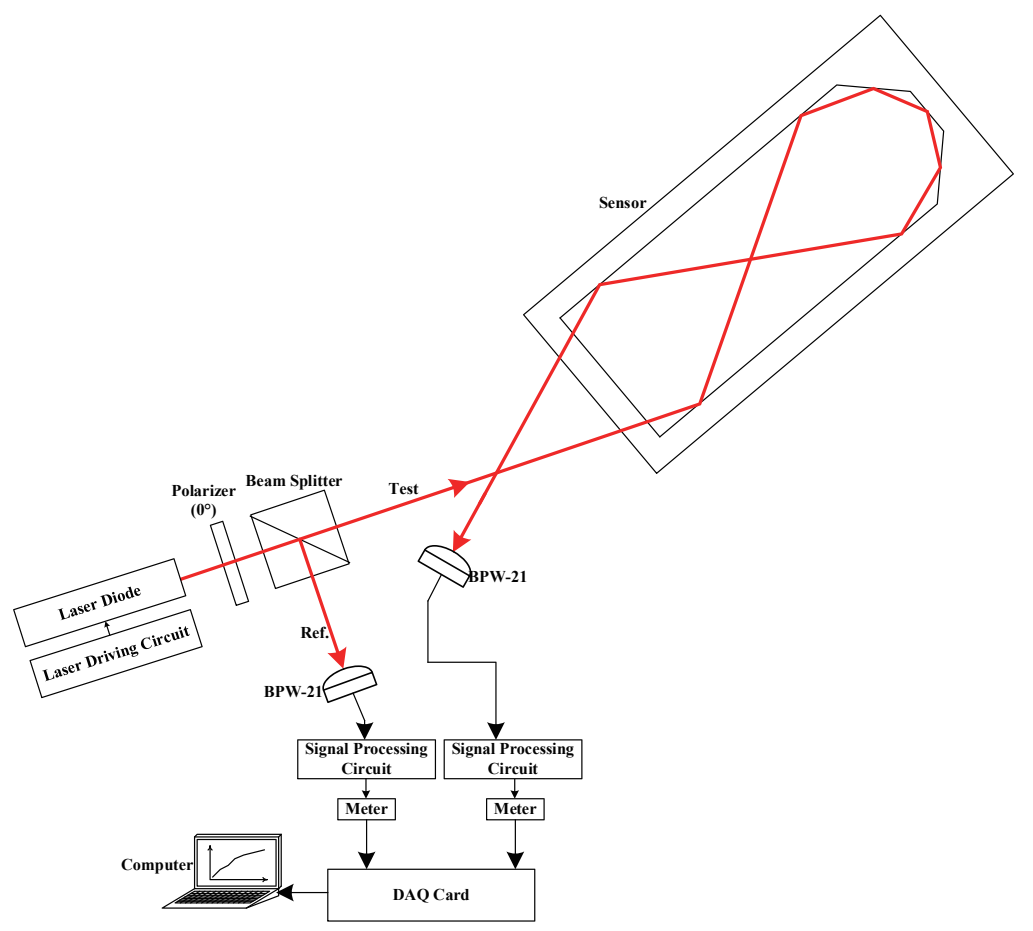

Fig. 5. (Color online) System architecture of the polygonal prism biochemical sensor.

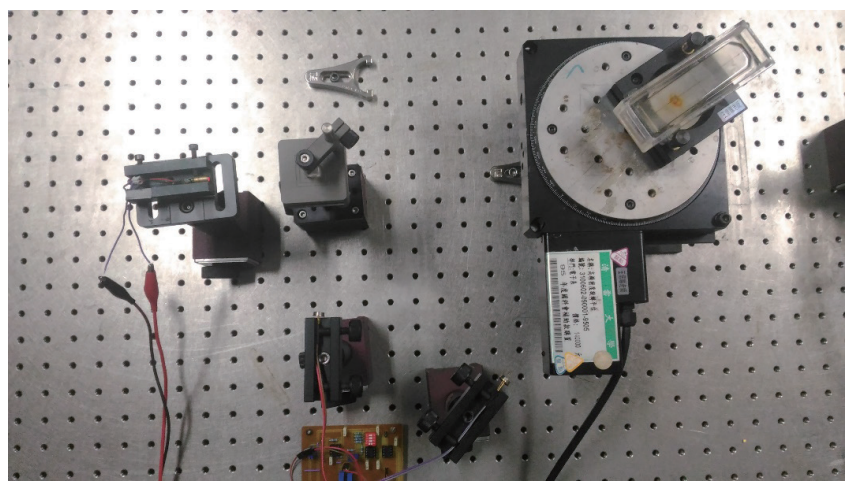

Fig. 6. (Color online) The photograph of the experimental setup.

\section{Experimental Results and Discussion}

In this experiment, ethanol at different concentrations (10-90\%) was used as the test fluid. As listed in Table 1, the refractive indices of alcohol with variable concentrations are measured with an Abbe refractometer (Model Type-1T.4T, Milton Roy Co.) at a temperature of $25^{\circ} \mathrm{C}$.

First, the polyhedral biosensor is placed in a sink and the alcohol of various concentrations is injected into the sink. Figure 7 shows the experimental and theoretical curves of the reflectivity $R_{p}$ of p-polarization light (TM wave) versus the refractive index of alcohole of 
Table 1

The refractive indices for alcohol of various concentrations using Abbe refractometer (Model Type-1T.4T, Milton Roy Co.) at temperature of $25^{\circ} \mathrm{C}$.

\begin{tabular}{cc}
\hline Concentration (\%) & Refractive index \\
\hline 0 & 1.3313 \\
10 & 1.332 \\
20 & 1.339 \\
30 & 1.342 \\
40 & 1.348 \\
50 & 1.353 \\
60 & 1.357 \\
70 & 1.359 \\
80 & 1.361 \\
90 & 1.3625 \\
\hline
\end{tabular}

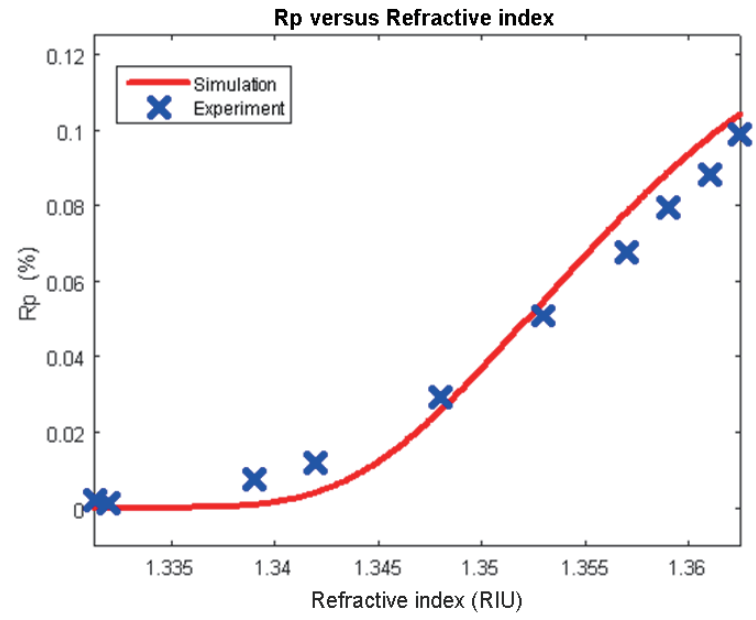

Fig. 7. (Color online) Experimental results and theoretical curve (Rp: reflectivity of TM wave).

various concentrations if the incidence angle is $71^{\circ}$ on surfaces $\mathrm{A}$ and $\mathrm{E}$. It can be seen that the experimental results and the theoretical curve are in good agreement. Therefore, the proposed optical system is feasible for fluid measurement.

The sensitivity $S$ of the sensor can be expressed as

$$
S=\frac{\partial R_{p}}{\partial n}
$$

where $\partial R_{p}$ is the reflectivity variation of p-polarization light and $\partial n$ represents changes in refractive indices. The relationship between refractive indices and sensitivity is shown in Fig. 8. In an optical system, high sensitivity is an important factor. Figure 8 shows that when the refractive index $n=1.3524$, the sensitivity can reach 6.1202 (\%/refractive index unit).

The resolution of this novel biosensor can be expressed as

$$
R=\frac{R_{m}}{S}
$$

where $R_{m}$ represents the resolution of the measuring device. Because the measuring device used in this study is a $4 \frac{1}{2}$ digital ammeter, the resolution of the meter, $R_{m}$, is equal to $0.005 \%$. Figure 9 shows the relationship between refractive indices and the resolution, indicating that the best resolution can reach $8.1 \times 10^{-6} \mathrm{RIU}$ when the refractive index is $n=1.3524$.

In the article, the thickness of metal films is the same as in the sensor of Wang et al. ${ }^{(7)}$ In fact, the thickness of metal films will affect the sensitivity. We try to simulate the sensitivity for different thicknesses of $\mathrm{Au}$ film with the thickness of Ti film at $2 \mathrm{~nm}$. In order to achieve better sensitivity and resolution, the thickness of Au film is in the range of $44-47 \mathrm{~nm}$. From Fig. 10, 


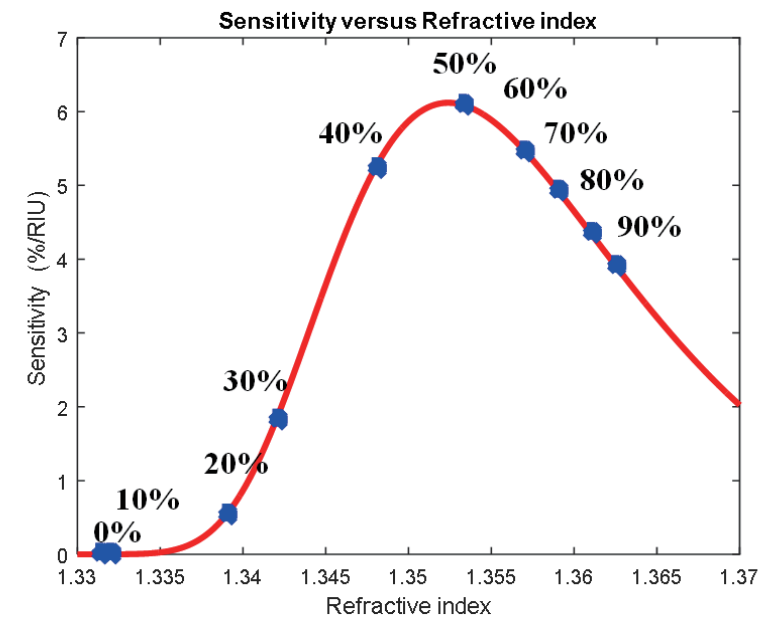

Fig. 8. (Color online) Relationship between sensitivity and refractive index in red light.

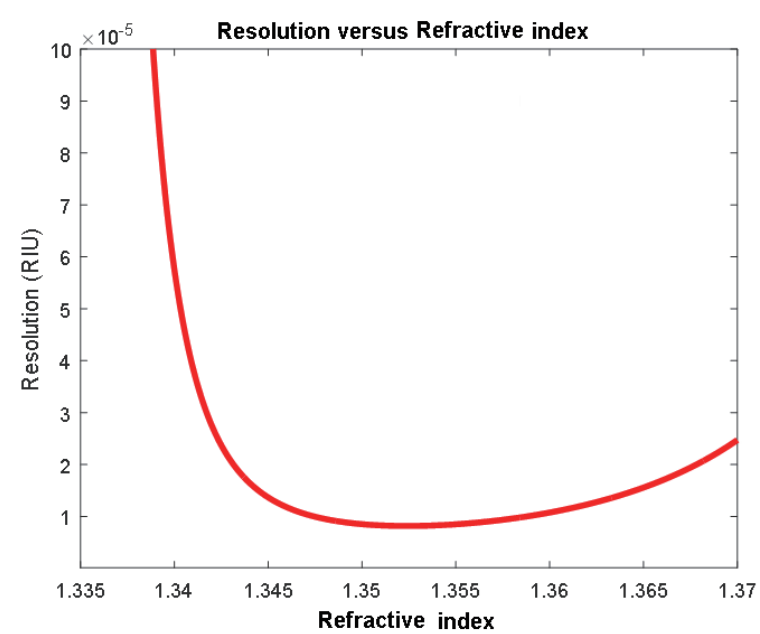

Fig. 9. (Color online) Relationship between resolution and refractive index in red light.

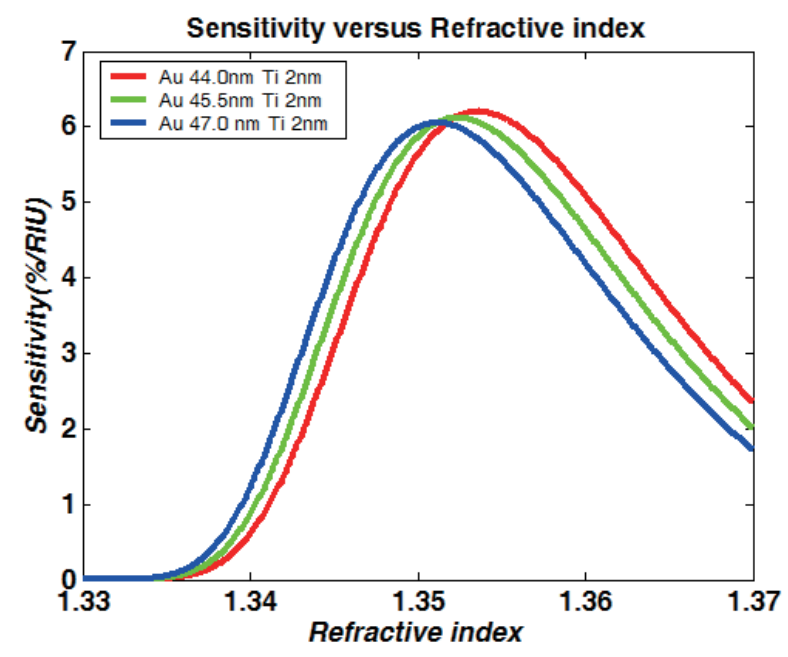

Fig. 10. (Color online) Au-film-thickness dependence of sensitivity.

it is evident that the sensitivity of the sensor with the Au-film thickness of $47 \mathrm{~nm}$ is better than those of the two others when the refractive index of the tested medium is smaller than 1.35 RIU. However, the sensitivity of the sensor with the Au-film thickness of $44 \mathrm{~nm}$ is better than those of the two others when the refractive index of the tested medium is larger than 1.35 RIU. For the reasons above, the Au-film thickness is chosen to be $45.5 \mathrm{~nm}$.

\section{Conclusion}

In this article, a polyhedral biosensor based on surface plasmon resonance technology was proposed. Owing to multiple ATRs in the biosensor, the sensitivity and resolution can be 
increased. With the polyhedral biosensor, the refractive index of the tested medium can be obtained only by measuring the intensity of p-polarization light exiting the sensor, owing to the effect of multiple ATRs. The sensing system has advantages such as a simple optical setup, easy operation, rapid measurement, and low cost.

In this study, the prism was coated with two layers of metal films: a layer of 2-nm-thick titanium and a layer of 45.5-nm-thick gold. The purpose of the $2 \mathrm{~nm}$ Ti film was to be an adhesion layer that allows the Au film to be closely coated on the prism. For simplification, the prism was coated with the 2-nm-thick Ti film in advance.

Although the best resolution of the phase method proposed by Wang et al. ${ }^{(7)}$ can reach $5.5 \times$ $10^{-7}$ RIU, which is better than the best resolution of $8.1 \times 10^{-6}$ RIU of the intensity method, the phase measurement method requires much more expensive equipment such as an electrooptic modulator and driver, lock-in amplifier, and arbitrary function generator. Additionally, the resolution of the polyhedral biosensor is better than that of the intensity method with the D-type fiber sensor presented by Wang et al. ${ }^{(11)}$ In the previous D-type fiber sensor, the best resolution can only reach $8 \times 10^{-5}$ RIU. In addition, the properties of the test substances are unaffected during the use of our sensor because no chemical reagents are needed. Therefore, our biosensor has great potential for future developments and could be valuable for chemical, biological, and biochemical sensing.

\section{Acknowledgments}

This research was partially supported by the Ministry of Science and Technology in Taiwan under Grant No. MOST 103-2221-E-231-006-.

\section{References}

1 P. Feifer, U. Aldinger, G. Schwotzer, S. Diekmann, and P. Steinrücke: Sens. Actuators, B 54 (1999) 166.

2 S. Jabbari, B. Dabirmanesh, S. S. Arab, M. Amanlou, S. Daneshjou, S. Gholami, and K. Khajeh: Sens. Actuators, B 240 (2017) 519.

3 A. K. Sharma, Rajan, and B. D. Gupta: Opt. Commun. 274 (2007) 320.

4 Rajan, S. Chand, and B. D Gupta: Sens. Actuators, B 123 (2007) 611.

5 A. K. Sharma and B. D. Gupta: Appl. Opt. 45 (2006) 151.

6 E. Oscar, D. H. Natalia, M. C. Navarrete, and G. C. Agustin: Appl. Opt. 45 (2006) 7294.

7 S. F. Wang, H. Ma, F. H. Kao, and A. L. Liu: Adv. Mater. Res. 716 (2013) 510.

8 E. Kretschmann: Z. Phys. 241 (1971) 313.

9 P. B. Johnson and R. W. Christy: Phys. Rev. B 6 (1972) 4370.

10 M. Born and E. Wolf: Principles of Optics (Pergamon Press, Oxford, 1970).

11 S. F. Wang, M. H. Chiu, J. C. Hsu, R. S. Chang, and F. T. Wang: Opt. Commun. 253 (2005) 283.

\section{About the Authors}

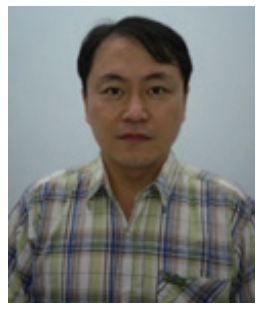

Jyh-Shayn Chiu received his M.S. degree from Chien Hsin University of Science and Technology, Jhongli, Taiwan, in 2007. He is currently pursuing a Ph.D. degree with the Department of Electrical Engineering, National Central University, Jhongli. His current research interests include photoelectric automation and plant factory automation measurement and testing. 


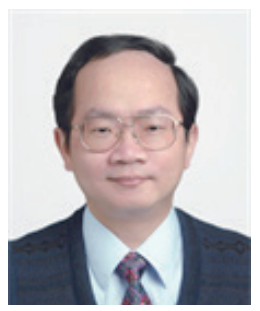

Shinn-Fwu Wang received his B.S. in electrophysics from National Chiao Tung University, Taiwan, in 1988, and his M.S. degree from the Institute of Optical Science, National Central University, Taiwan, in 1990. He began work toward a Ph.D. degree in optical metrology at the Institute of Optical Science, National Central University in September 2002, and received his $\mathrm{Ph}$. D. in January 2005. He joined the faculty of the Department of Electronic Engineering at Chien Hsin University of Science and Technology in 1991. He became a full professor in 2009. His current research interests are in the areas of optical metrology, optical images processing, biosensors, fiber optic sensors, LED lighting optics, LED plant factory, display optics, biosensing systems, microcontrollers, and smart structures.

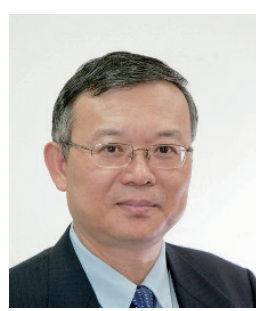

Wen-June Wang received his B.S. degree from the Department of Control Engineering, National Chiao-Tung University, Hsin-Chu, Taiwan, in 1980, and his M.S. degree from the Department of Electrical Engineering, Tatung University, Taipei, Taiwan, in 1984. He received his Ph.D. degree from the Institute of Electronics, National Chiao-Tung University of Taiwan in 1987. Professor Wang is presently a Chair Professor of the Department of Electrical Engineering. He was the Dean of the College of Electrical Engineering and Computer Science, National Central University, Chung-Li City, Taiwan. He was also a Chair Professor and the Dean of the Research and Development Office of National Taipei University of Technology, Taipei, Taiwan, from 2007 to 2009. From 2005 to 2007, he was the Dean of the College of Science and Technology, National Chi-Nan University, Puli, NanTou, Taiwan. In addition, Professor Wang became an IEEE Fellow in 2008. Professor Wang has authored or coauthored over 150 reviewed journal papers and 150 conference papers in the areas of fuzzy systems and theorems, robust and nonlinear control in large-scale systems, and neural networks. His most significant contributions are in the design of fuzzy systems and the development of robotics. His other research interests include robot control, neural networks, and pattern recognition. 